\title{
Microscopic Pendulum Modelling: Absurd Idea or Innovative View on Old Problems?
}

\author{
Martin Bicher ${ }^{1 *}$, Niki Popper ${ }^{2}$ \\ ${ }^{1}$ Institute for Analysis and Scientific Computing, TU Wien, Wiedner Hauptstraße 8-10, \\ 1040 Vienna, Austria; ${ }^{*}$ martin.bicher@tuwien.ac.at \\ ${ }^{2}$ dwh Simulation Services, dwh GmbH, Neustiftgasse 57-59, 1070 Vienna, Austria
}

SNE 27(4), 2017, 177-182, DOI: 10.11128/sne.27.tn.10392 Received: October 20, 2017, Revised: December 3, 2017, Accepted: December 10, 2017

SNE - Simulation Notes Europe, ARGESIM Publisher Vienna, ISSN Print 2305-9974, Online 2306-0271, www.sne-journal.org

Abstract. Especially in the last years the idea of finding a simpler meta-model for a developed simulation model has become more and more popular as not only the research questions, but also the resulting models have become more and more complex. The meta-model hereby helps understanding the behaviour of the original model and supports its validation and verification process. Moreover, it also gives a second perspective on the modelled system. Mean-field theory is a very formal link between microscopic and macroscopic models and can be used to find meta-models for either of the two types. Usually so-called mean-field analysis is used to find a summarising simpler macroscopic model for a given complex microscopic approach, but we will emphasise the inverse process in this work: Applying inverse mean-field analysis on an ordinary differential equation model we systematically derive a microscopic representation of the model. On purpose we chose a very unusual model to apply the method to: the un-damped linearised pendulum equation. Hereby we want to emphasise the method's flexibility and generality, investigate possible benefits of the gained microscopic meta-model for this strange field of application, and discuss about interpretation of the elements of the resulting agent-based pendulum model.

\section{Introduction}

According to German philosopher Herbert Stachowiak [1] any model, in general, is defined as a (usually) simplified image of a real or artificial object that has been developed for a specific purpose and time of usage.
This definition clearly permits the idea of developing a model $M_{2}$ for a different model $M_{1}$, a so-called meta-model, developed for a specific purpose related to $M_{1}$. For simulation models this strategy becomes more and more popular nowadays due to steadily increasing complexity of research questions and models. Hereby the more simplified meta-model $M_{2}$ can be used to either get insights into $M_{1}$ to simplify its verification and validation process (Compare with Bernhard Zeigler's talk "Why Should We Develop Simulation Models in Pairs" at WSC 2017 [2]) or to get new ideas about the modelled system.

Mean-Field Approximation (MFA) is one concept for finding such a meta-model for a given microscopic model. Hereby chosen aggregated numbers of the microscopic model $\left(M_{1}\right)$ are approximated by solutions of specific ordinary or partial differential equations - the so-called mean-field model $\left(M_{2}\right)$. For detailed information the reader is referred to [3]. One key advantage of this method is that the meta-model is (in principle) found by a formal recipe that guarantees the "correctness" of the meta-model with respect to (asymptotic) equivalence of the model results. The key to this equivalence lies within a formal theorem, generally referred to as Mean-Field Theorem (MFT), which states how the elements of the microscopic model need to be used to find in macroscopic equation-based meta-model, the so-called mean-field model. The formality of this procedure makes the strategy extremely generic and completely independent of the investigated model's field of application. It can e.g. be applied to find mean-field models for agent-based models in economics [4] or sociology [5] as well as for cellular automata models in epidemiology [6, 7] or population dynamics [8].

Another example that emphasises the generality of the method is gained interpreting mean-field approximation as an invertible mapping from a microscopic to a mean-field model. Inverse MFA is presented in [3], Chapter 5.2.3, on the example of the Levins Model 
[9], a macroscopic model for the growth of a population. Using this inverse strategy, a microscopic representation of this non-linear ordinary differential (ODE) model is derived systematically. Hereby the role of the meta-model $M_{2}$ is switched from the macroscopic to the microscopic one. Although a meta-model in form of a microscopic approach, e.g. in form of an agent-based model, can hardly be considered particularly easier than the original macroscopic one, it is definitely more flexible and avoids specific problems with the numerics of e.g. ODE solvers. Moreover, it gives a very interesting view on the original system as the microscopic elements can be interpreted as single agents.

In this work we want to apply inverse MFA on a much more abstract example: the linearised, undamped pendulum model given by

$$
\ddot{\varphi}(t)=-k \varphi(t), \varphi(0)=\varphi_{0}, \dot{\varphi}(0)=0,
$$

with $k>0$. Although, in the contrary to the Levins model, there is no feasible justification for modelling a pendulum in a microscopic way we will apply this formal method to

a) emphasise its flexibility and generality by showing that it actually works,

b) investigate possible benefits of the gained microscopic meta-model for this particular application, and

c) attempt to interpret the microscopic elements of the resulting meta-model.

\section{Inverse Mean-Field Approximation}

In order to perform the mentioned inverse MFA we will stress the strategy proposed in [3] Chapter 4.6.4. Key idea of this strategy is finding suitable transformations, so that the equation of the ODE model has the form of the system

$$
\begin{gathered}
\frac{d \vec{Y}_{1}}{d t}=\sum_{j=1}^{d} \vec{Y}_{j} \omega_{j, 1}(t, \vec{Y})-\vec{Y}_{1} \omega_{1, j}(t, \vec{Y}) \\
\vdots \\
\frac{d \vec{Y}_{d}}{d t}=\sum_{j=1}^{d} \vec{Y}_{j} \omega_{j, d}(t, \vec{Y})-\vec{Y}_{d} \omega_{d, j}(t, \vec{Y})
\end{gathered}
$$

of ordinary differential-equations (ODEs) with parameter functions $\omega_{i, j} \geq 0, i, j \in\{1, \ldots, d\}$ and sufficiently large positive solutions $\vec{Y}(t)$. In case such a representation has been found, the parameter functions $\omega$ can be used as transition rates of the microscopic elements of a microscopic model. Hereby $\omega_{i, j}$ denotes the rate (transition-rate) that an individual with state $i$ switches to state $j$. Let $a_{k}(t) \in\{1, \ldots, d\}$ denote the time dependent state of individual $k$ in the newly defined microscopic model with $N$ individuals in total, then (a version of) the Mean-Field Theorem (Corollary 5.1 in [3]) states that the aggregated numbers

$$
\vec{X}_{i}(t):=\sum_{k=1}^{N} \mathbb{1}_{i}\left(a_{k}(t)\right), \quad i \in\{1, \ldots, d\}
$$

fulfil $\vec{X}_{i}(t)=\vec{Y}_{i}(t)+\mathscr{O}(\sqrt{N})$, wherein $\vec{Y}$ is the solution of above system of ODEs. Note, that $\vec{X}$ is precisely the total number of microscopic elements that share the same state at time $t$. In case $\omega_{i, j}$ depends on $\vec{Y}$ in the ODE system, the individual transition rates depend on the aggregated number vector $\vec{X}$ accordingly. The latter can be interpreted as interaction of the microscopic elements on the aggregated level.

In [3] Chapter 4.6.4 a step-by-step instruction gives hints how mentioned transformation can be found. We will mainly follow these proposed steps:

a) First of all, the pendulum equation, a second order ODE, needs to be written as a system of first order ODEs via

$$
\varphi(t)^{\prime \prime}=-k \varphi(t) \Rightarrow\left(\begin{array}{c}
\varphi \\
\psi
\end{array}\right)^{\prime}(t)=\left(\begin{array}{c}
\psi(t) \\
-k \varphi(t)
\end{array}\right)
$$

b) Moreover, the variable-functions appearing in (2) need to be positive which is violated in (4) as angle and angular velocity resulting from the pendulum equation might also have negative values. Therefore, we need to translate the equation via

$$
\left(\begin{array}{c}
\tilde{\varphi} \\
\tilde{\psi}
\end{array}\right):=\left(\begin{array}{c}
\varphi \\
\psi
\end{array}\right)+T
$$

so that $\tilde{\varphi}, \tilde{\psi}$ is always positive. For physically feasible systems this translation is always possible as model trajectories need to be stable. In case of the pendulum it is easy to reason with physical arguments (e.g. Newton's axioms) that $\varphi(t) \in\left[-\left|\varphi_{0}\right|,\left|\varphi_{0}\right|\right]$ and $\dot{\varphi}(t) \in\left[-\sqrt{k}\left|\varphi_{0}\right|, \sqrt{k}\left|\varphi_{0}\right|\right]$ and therefore any

$$
T>\max \left\{\sqrt{k}\left|\varphi_{0}\right|,\left|\varphi_{0}\right|\right\}
$$


is sufficiently large to guarantee that

$$
(4) \Longrightarrow \quad\left(\begin{array}{c}
\tilde{\varphi} \\
\tilde{\psi}
\end{array}\right)^{\prime}(t)=\left(\begin{array}{c}
\tilde{\psi}(t)-T \\
-k \tilde{\varphi}(t)+k T
\end{array}\right),
$$

has positive solutions $\tilde{\varphi}$ and $\tilde{\psi}$.

c) As the variable-functions will end up approximating the aggregated numbers of a microscopic model, we need to rescale the equation to get them sufficiently large. We define

$$
\left(\begin{array}{c}
\bar{\varphi} \\
\bar{\psi}
\end{array}\right):=S\left(\begin{array}{c}
\tilde{\varphi} \\
\tilde{\psi}
\end{array}\right),
$$

leading to

$$
(6) \Longrightarrow\left(\frac{\bar{\varphi}}{\bar{\psi}}\right)^{\prime}(t)=\left(\begin{array}{c}
\bar{\psi}-S T \\
-k \bar{\varphi}+k S T
\end{array}\right) .
$$

d) As (2) conserves the sum

$$
\sum_{i=1}^{d} Y_{i}^{\prime}(t)=0
$$

we introduce a third state $\bar{\xi}$ and write

$$
\text { (7) } \Rightarrow\left(\begin{array}{c}
\bar{\varphi} \\
\bar{\psi} \\
\xi
\end{array}\right)^{\prime}=\left(\begin{array}{c}
\bar{\psi}-S T \\
-k \bar{\varphi}+k S T \\
k \bar{\varphi}-k S T-\bar{\psi}+S T
\end{array}\right) \text {. }
$$

Hereby we need to care for a feasible initial condition. As the sum of the right-hand-sides is zero, the sum of the variable functions is constant $\bar{\varphi}+\bar{\psi}+\bar{\xi}=C$. As $0<\bar{\varphi}<2 S T$ and $0<\bar{\psi}<2 S T$ positivity for $\bar{\xi}$ is ensured if (e.g.) $\bar{\xi}_{0}:=4 S T$.

e) As the transition rates $\omega$ need to be positive functions, we need to rewrite the above modified pendulum equation one last time to finally match the form of (2):

$$
\begin{gathered}
(8) \Longrightarrow \\
\left(\begin{array}{c}
\bar{\varphi} \\
\frac{\bar{\psi}}{\xi}
\end{array}\right)^{\prime}=\left(\begin{array}{c}
\frac{\max (\bar{\psi}-S T, 0)}{\bar{\xi}} \bar{\xi} \\
\frac{\max (-k \bar{\varphi}+k S T, 0)}{\bar{\xi}} \bar{\xi} \\
\frac{\max (-\bar{\psi}+S T, 0)}{\bar{\varphi}} \bar{\varphi}+\frac{\max (k \bar{\varphi}-k S T, 0)}{\bar{\psi}} \bar{\psi}
\end{array}\right)
\end{gathered}
$$

$$
-\left(\begin{array}{c}
\frac{\max (-\bar{\psi}+S T, 0)}{\bar{\varphi}} \bar{\varphi} \\
\frac{\max (k \bar{\varphi}-k S T, 0)}{\bar{\psi}} \bar{\psi} \\
\frac{\max (\bar{\psi}-S T, 0)+\max (-k \bar{\varphi}+k S T, 0)}{\bar{\xi}} \bar{\xi}
\end{array}\right) .
$$

The last transformation (9) finally makes it possible to match (2). We identify and define (for $\bar{\varphi} \rightarrow \vec{X}_{1}, \bar{\psi} \rightarrow$ $\left.\vec{X}_{2}, \bar{\xi} \rightarrow \vec{X}_{3}\right)$

$$
\left(\begin{array}{l}
\omega_{1,3} \\
\omega_{2,3} \\
\omega_{3,1} \\
\omega_{3,2}
\end{array}\right)(t, \vec{X}):=\left(\begin{array}{c}
\frac{\max \left(-\vec{X}_{2}+S T, 0\right)}{\bar{X}_{1}} \\
\frac{k \max \left(\bar{X}_{1}-S T, 0\right)}{\bar{X}_{2}} \\
\frac{\max \left(\bar{X}_{2}-S T, 0\right)}{\bar{X}_{3}} \\
\frac{k \max \left(-\bar{X}_{1}+S T, 0\right)}{\bar{X}_{3}}
\end{array}\right) .
$$

Using these transition rates in a microscopic model, the aggregated numbers of this model with asymptotically equal the solutions of (9). As

$$
\overline{1}\left(\begin{array}{l}
\bar{\varphi} \\
\bar{\psi} \\
\xi
\end{array}\right)-T=\left(\begin{array}{l}
\varphi \\
\psi \\
\xi
\end{array}\right)
$$

an affine transformation (scale $S$ and transition $T$ ) of the aggregated numbers of the first state of the microscopic model approximates the solution of the pendulum equation, the angle of the pendulum. The aggregated number of the second state corresponds to the angular velocity and will also be regarded in our analysis.

Following [3] we introduce a time-step size $d t$ to set up a time-discrete microscopic model. Therefore, for all microscopic elements $a_{k}$ of the new model, we deduce the transition probabilities

$$
P\left(a_{k}(t+d t)=i \mid a_{k}(t)=j, \vec{X}\right):=d t \cdot \omega_{j, i}(t, \vec{X}) .
$$

This can be interpreted as a first order Euler (or EulerMaruyama see [10]) approximation.

\section{Agent-Based Pendulum Model}

Given by the results of the last section, the basis for a microscopic meta-model was developed for the undamped pendulum model. To simplify speech, we consider the established model as an agent-based model $(\mathrm{ABM})$ :

Model 1 (Agent-Based Pendulum Model). With $\varphi_{0}$ and $\dot{\varphi}_{0}$ denoting the initial conditions of the pendulum equation and $k$ the frequency parameter, we define the fol- 
lowing microscopic model via its initialisation phase and its time dynamics.

\section{Initialisation:}

- Define a translation parameter $T>$ $\max \left\{\sqrt{k}\left|\varphi_{0}\right|,\left|\varphi_{0}\right|\right\}$ and a sufficiently large scale parameter $S \in \mathbb{R}^{+}$(usually $S \gg 100$ makes sense).

- Define a time-step length dt small enough to guarantee that the probabilities used in the dynamics below are feasible (i.e. smaller than 1).

- Moreover, calculate

$$
\begin{aligned}
& X_{1}:=\left\lfloor\overline{\varphi_{0}}\right\rfloor=\left\lfloor S\left(\varphi_{0}+T\right)\right\rfloor \\
& X_{2}:=\left\lfloor\overline{\psi_{0}}\right\rfloor=\lfloor S T\rfloor, \text { and } \\
& X_{3}:=\left\lfloor\overline{\xi_{0}}\right\rfloor=\lfloor 4 S T\rfloor .
\end{aligned}
$$

- Setup a model with $N:=X_{1}+X_{2}+X_{3}$ agents of which each is assigned one of three states, henceforth denoted as angle (state 1), velocity (state 2) and idle (state 3). Assign $X_{1}$ agents in state 1, $X_{2}$ and $X_{3}$ agents in state 2 and 3 accordingly. The total number of agents in any of the three states is tracked throughout the model using these three variables $X_{1}, X_{2}$ and $X_{3}$.

\section{Dynamics:}

- The model is updated in equidistant time-steps of length $d t$.

- Each time-step, each agent is addressed once. According to its prior state, specific probabilities decide if the agent's state remains or changes.

- In case an agent is in the angle-state and $\vec{X}_{2} \leq S T$, it may switch to the idle-state with probability

$$
d t \cdot \omega_{1,3}=d t \frac{S T-X_{2}}{X_{1}} .
$$

Otherwise, it remains in its state. In case of a switch, $\vec{X}_{3}++$ and $\vec{X}_{1-}$.

- In case an agent is in the velocity-state and $\vec{X}_{1} \geq$ $S T$, it may switch to the idle-state with probability

$$
d t \cdot \omega_{2,3}=d t \frac{X_{1}-S T}{X_{2}} .
$$

Otherwise, it remains in its state. In case of a switch, $\vec{X}_{3}++$ and $\vec{X}_{2}-$.
- In case an agent is in the idle-state and $\vec{X}_{2}>S T$, it may switch to the angle-state with probability

$$
d t \cdot \omega_{3,1}=d t \frac{X_{2}-S T}{X_{3}}
$$

or, in case $\vec{X}_{1}<S T$, to the velocity-state with probability

$$
d t \cdot \omega_{3,2}=d t \frac{S T-X_{1}}{X_{3}}
$$

Otherwise, it remains in its state. In case of a switch, change the aggregated numbers $X_{1}, X_{2}$ and $X_{3}$ accordingly.

The flow-chart in Figure 1 shows how the agents may change their states: They may switch from states "angle" and "velocity" to state "idle" and vice versa. They never switch between "angle" and "velocity" directly.

Moreover, the concept that the transition probabilities of this model depend on the aggregated numbers can be translated as a kind of global-level-interaction between the agents. It is comparable with an economical model of the stock-market: the stock-price is a globally known variable that influences the buying behaviour of all actors. Yet it changes in response to transactions, i.e. to individual behaviour. In the Levins Model presented in [3] in Chapter 5.2.3 the author referred the resulting global-level-interactions in this model to local-level-interactions - i.e. typical agentagent contacts - via stochastic equivalences comparable to picking balls from urns. In this case, we did not attempt to find such a representation as it would be too complicated (but surely not impossible). Extensive use of the Bayesian Theorem and sufficient creativity would be required.

According to [3] the aggregated numbers of above model asymptotically equal the solutions of the affine transformed pendulum equation for

- $S \rightarrow \infty$ (equivalent to $N \rightarrow \infty$ ) and

- $d t \rightarrow 0$.

Figure 2 shows a series of convergence-tests for different scaling parameters $S$ and time-step parameters $d t$. Blue and red lines show the aggregated results $X_{1}$ and $X_{2}$ of sample runs of the ABM. The black and green line show the scaled and translated analytic solutions $\overline{\varphi(t)}=$ $S(T+\cos (\sqrt{2} t))$ and $\overline{\psi(t)}=S(T-\sqrt{2} \sin (\sqrt{2} t))$ of the pendulum equation. For large time-steps sizes $d t$ the ABM model results becomes unstable similar to explicit 
Runge-Kutta methods for the solution of ODEs. Yet, the convergence properties can be verified.

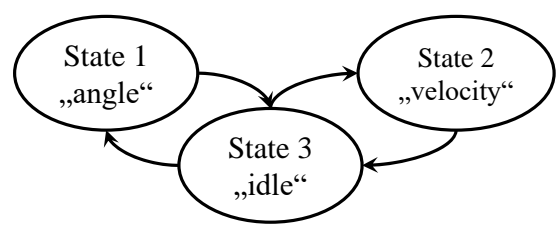

Figure 1: Flowchart of the agent-based model 1.

\section{Discussion}

The presented strategy leaves three interesting aspects for discussion.

Discussion of the Agent-Based Model. Clearly, the most interesting question concerning the derived model is, whether the model is not only a meta-model for the pendulum equation, but also a model for the real pendulum. According to Stachoviak [1] (compare with Section Introduction) it would be necessary to identify the agent-based model as a simplified image of a real pendulum.

Though undoubtedly consisting of microscopic elements like molecules or atoms, a pendulum (say, a rope pendulum with a sphere-shaped weight) is in principle not a system that is modelled as the sum of its microscopic elements. As the sphere is usually assumed to be made of a solid material, interactions between the elements are very limited and hardly contribute to the total movement of the object. Moreover, any molecule/atom in the swinging ball has an angle and a velocity and is not either "angle" or "velocity". If any, probably only a quantum-mechanic interpretation is somehow legitimate: Say one particle represents a fictive sub-atomic particle that is responsible for one quantum of angle and one quantum of velocity. If excited to either of the two states it results the object it is "attached" either to move or to accelerate. The chances for excitation and relaxation are directly linked to the laws of total energyconservation. Yet, as this interpretation is very vague, we would not really consider Model 1 as a model of the real pendulum, but solely as a meta-model.

Instead, we would suggest interpreting the derived model as a "numerical solver" of the pendulum equation. Using a microscopic solver on a macroscopic, equation based model is not unusual and is commonly
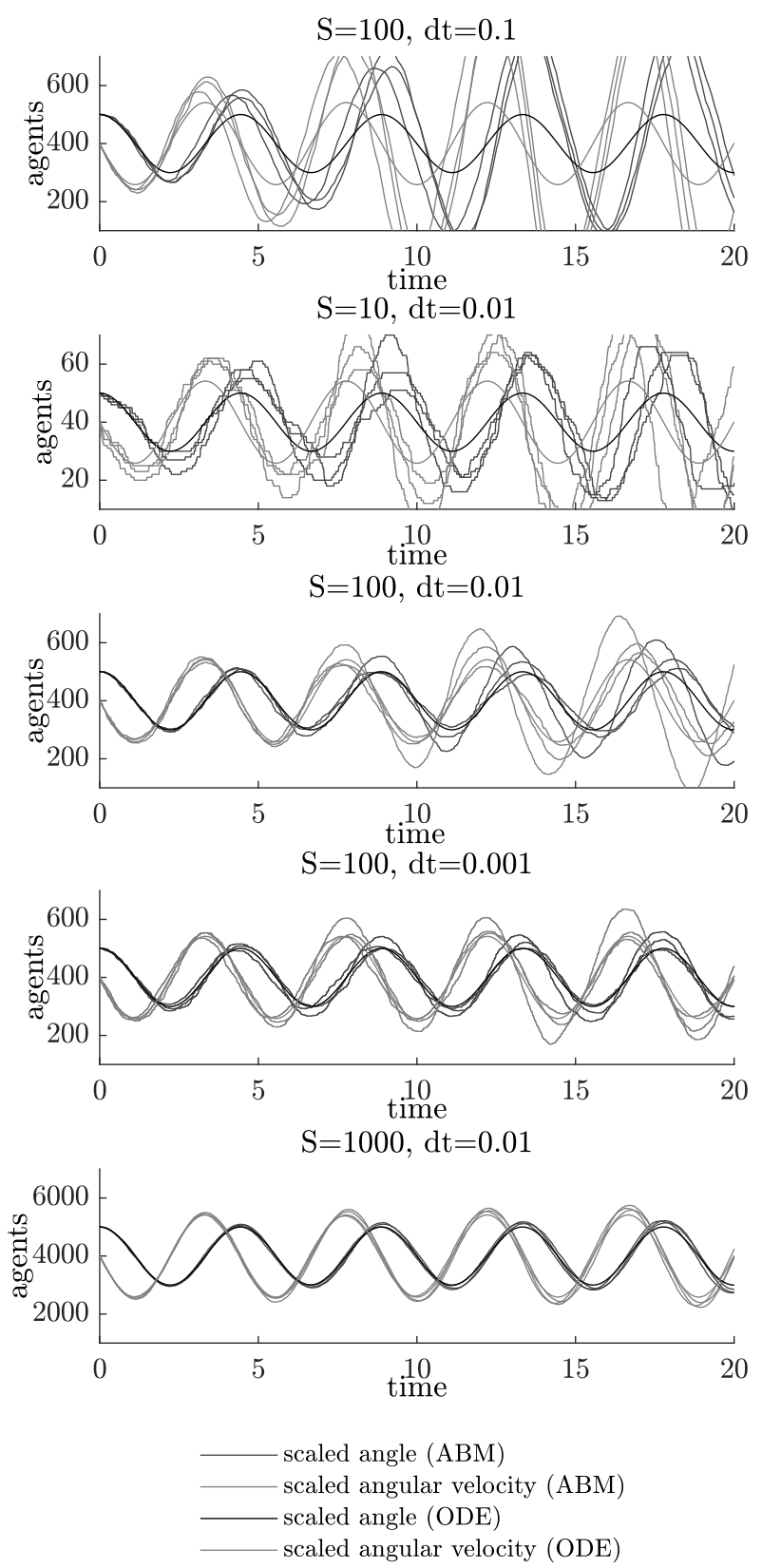

Figure 2: Tests for different values of $S$ and $d t$ for the stochastic ABM (3 runs each) defined in Model 1 for $\varphi_{0}=\frac{\pi}{2}, k=2$ and $T=4$. 
done e.g. in fluid dynamics: The macroscopic NavierStokes equations are "discretised" by particles on a grid (see HPP, FHP and Lattice-Boltzmann method [11]).

Discussion of the Benefits of the Model. The popularity of mentioned microscopic modelling approaches in fluid-dynamics simulation is mainly a result of their flexibility: Not only does a particle approach allow almost arbitrarily complex boundary-geometry (posing difficulties for a PDE approach with classic Navier-Stokes Equations), it can also be applied easily to simulate heterogeneous liquids. In general, microscopic models are clearly the most flexible of all model types with respect to model extensions and modifications due to their object-oriented description.

We observe similar flexibility for the microscopic pendulum model introducing a state-event: While stateevents are trivially detected in the microscopic approach (it is quantised) ODE solvers often need to iterate to find them adjusting their step-size.

Discussion of the Inverse Mean-Field Method. Apart from the derived model itself, the example of the pendulum model emphasises the generality of the inverse mean-field approximation for finding microscopic meta-models to macroscopic systems. Although there is no obvious reason for a microscopic representation of the system in that particular case the method definitely leads to a different point-of-view of the real system. R.E. Shannon, one of the founding-fathers of systems simulation, emphasised in 1975 that not only the measurable evaluation of various designs of a given system is target of a simulation model, but also the understanding of its behaviour [12]. Hence analysis of effects of different system components poses a vital contribution to any given research problem. The availability of a microscopic and a macroscopic model for one system allows two different points of view on the system components.

\section{Acknowledgement}

K-Projekt DEXHELPP is supported by BMVIT, BMWFW and the state of Vienna via COMET - Competence Centers for Excellent Technologies. Programme COMET is processed by FFG.

\section{References}

[1] Stachowiak H. Allgemeine Modelltheorie. Wien, New York: Springer-Verlag. 1973.

[2] Zeigler B. Why Should We Develop Simulation Models in Pairs? 2017.

URL https:

/ / www youtube.com/watch?v=iGXLxZcpUjA

[3] Bicher M. Classification of Microscopic Models with Respect to Aggregated System Behaviour. Dissertation, TU Wien, Vienna, Austria. 2017.

[4] Aoki M. Modeling aggregate behavior and fluctuations in economics: stochastic views of interacting agents. New York: Cambridge University Press. 2002.

[5] Deffuant G, Neau D, Amblard F, Weisbuch G. Mixing beliefs among interacting agents. Advances in Complex Systems. 2000;03(01n04):87-98.

[6] Webb SD, Keeling MJ, Boots M. Host-parasite interactions between the local and the mean-field: How and when does spatial population structure matter? Journal of Theoretical Biology. 2007;249(1):140-152.

[7] Benoit J, Nunes A, Telo da Gama M. Pair approximation models for disease spread. The European Physical Journal B. 2006;50(1-2):177-181.

[8] Morita S, Tainaka Ki. Undamped oscillations in prey-predator models on a finite size lattice. Population Ecology. 2006;48(2):99-105.

[9] Levins R. Some Demographic and Genetic Consequences of Environmental Heterogeneity for Biological Control. Bulletin of the Entomological Society of America. 1969;15(3):237-240.

[10] Kloeden PE. Numerical solution of stochastic differential equations. No. 23 in Applications of mathematics. Berlin ; New York: Springer, corr. 3rd print ed. 1999.

[11] Frisch U, Hasslacher B, Pomeau Y. Lattice-Gas Automata for the Navier-Stokes Equation. Physical Review Letters. 1986;56(14):1505-1508.

[12] Shannon RE. Systems Simulation: The Art and Science. Englewood Cliffs, N.J: Prentice-Hall. 1975. 